hep-th/0410054

UCLA/04/TEP/42

SPhT-T04/125

PUPT-2137

NSF-KITP-04-112

\title{
Loops in Twistor Space
}

\author{
Iosif Bena and Zvi Bern \\ Department of Physics and Astronomy \\ University of California, Los Angeles, Calif. 90095-1547 \\ iosif, bern@physics .ucla.edu \\ David A. Kosower \\ Service de Physique Théorique ${ }^{\natural}, C E A-S a c l a y$ \\ F-91191 Gif-sur-Yvette cedex, France \\ kosower@spht.saclay.cea.fr \\ and \\ Radu Roiban \\ Department of Physics \\ University of California, Santa Barbara, Calif. 93106 \\ and \\ Department of Physics \\ Princeton University, Princeton, New Jersey 08544 \\ rroiban@princeton.edu
}

\begin{abstract}
We elucidate the one-loop twistor-space structure corresponding to momentum-space MHV diagrams. We also discuss the infrared divergences, and argue that only a limited set of MHV diagrams contain them. We show how to introduce a twistor-space regulator corresponding to dimensional regularization for the infrared-divergent diagrams. We also evaluate explicitly the 'holomorphic anomaly' pointed out by Cachazo, Svrček, and Witten, and use the result to define modified differential operators which can be used to probe the twistor-space structure of one-loop amplitudes.
\end{abstract}

${ }^{\natural}$ Laboratory of the Direction des Sciences de la Matière of the Commissariat à l'Energie Atomique of France. 


\section{Introduction}

In a recent paper [1], Witten suggested that tree-level amplitudes of non-Abelian gauge theories can be obtained by integrating over the moduli space of certain $D$-instantons in the openstring topological B-model on (super) twistor space $\mathbb{C P}^{3 \mid 4}$. This proposal generalizes Nair's earlier construction [2] of maximally helicity-violating (MHV) amplitudes [3,4]. It may help shed light on the relative simplicity of amplitudes in unbroken gauge theories. Cachazo, Svrček, and Witten (CSW) [5] distilled the twistor-space structure underlying gauge-theory amplitudes in Witten's proposal into a novel method to construct arbitrary tree-level amplitudes, using off-shell continuations of MHV amplitudes. Although no-one has yet given a direct derivation of the CSW construction from a Lagrangian, the combination of the correct pole structure and Lorentz invariance leaves little doubt that it is correct. Related investigations and applications of the CSW construction have appeared in refs. $[6,7,8]$.

The success of the twistor-space picture at tree level leads one to investigate whether it can be extended to loop corrections. In the twistor-space string theory (or theories*) proposed by Witten [1], Berkovits [9], and Neitzke and Vafa [10], loop corrections appear to include conformal supergravitons in an inextricable way. (Siegel [11] has proposed an alternative approach.) However, a more straightforward and direct approach to this question was proposed by Brandhuber, Spence, and Travaglini (BST) [12]. They were able to map one-loop diagrams built out of two MHV vertices to the correct one-loop result for MHV amplitudes [13], by showing that their cuts map directly onto the cuts used in calculating the amplitudes. We will call such diagrams twistor-rules diagrams. (A more direct calculation of such loop diagrams without reference to the cuts fails to give the correct answer, presumably because of lack of a correct $i \varepsilon$ prescription.) Abe, Nair, and Park's construction [14] of an effective Lagrangian points in the same direction.

The BST calculation does not yet have a twistor-space derivation, but it may be taken as 'experimental' evidence that such a derivation should exist. This leads to the question: what is the twistor-space picture corresponding to their calculation? At tree level, the twistor-space picture corresponding to the CSW construction takes sets of points on various lines (each corresponding to one MHV vertex), and connects these lines by twistor-space propagators in such a way as to obtain a connected tree-level topology. At tree level, one can further argue [15] that this construction is equivalent to a picture in which all propagators collapse to points, forcing the attached lines

\footnotetext{
* It is not clear to us that the three theories discussed in refs. $[1,9,10]$ are in fact identical.
} 
to intersect. This confirms the original observation of Cachazo, Svrček, and Witten, based on known results for the amplitudes, that a tree-level twistor-space amplitude is supported on a locus of intersecting lines. Gukov et al. also argue that this construction is then equivalent to one in which the points lie on a higher-degree curve in twistor space. (Three of the authors presented a field-theory picture of this equivalence [7].) This matches the calculations done by Spradlin, Volovich, and one of the authors [16], and explains why the one-instanton calculation is equivalent to a disconnected multi-instanton one.

The explicit calculations suggest that in twistor space, one-loop amplitudes are supported on lines connected by propagators, the ensemble forming a connected genus-one topology. We will show that the BST form matches this intuition. This may not seem to be in accord with the configurations found by Cachazo, Svrček, and Witten in their paper on MHV loop amplitudes [17]. While they found that the infrared-divergent contributions are supported precisely on configurations of two lines connected by propagators, it appeared that the finite parts are supported on configurations where one propagator has collapsed, and one other point has wandered off its original line into the plane defined by the two intersecting lines. As the same authors have argued more recently [18], however, there is a subtlety in the use of momentum-space differential operators to establish this picture. We will compute explicitly the 'holomorphic anomaly' they point out, and show that when it is taken into account, the twistor-space picture of the amplitudes is indeed the one suggested by the twistorspace partner to the BST calculation as presented herein. One can define modified differential operators, subtracting the anomaly contribution, which do annihilate the one-loop amplitude.

In the next section we review in detail the relation between a tree-level momentum-space amplitude and the corresponding twistor-space amplitude. Before following a similar path for oneloop amplitudes, we must understand where their infrared divergences emerge; we do so in sect. 3. We then give the twistor-space counterpart of infrared-finite twistor-rules diagrams in sect. 4, and the infrared-divergent ones in sect. 5. We also show how to regulate the divergences in twistor space in a manner compatible with ordinary dimensional regularization in momentum space. In sect. 6, we compute the 'holomorphic anomaly' explicitly, and show that one can use it to define a modified differential operator for probing the twistor-space structure of amplitudes. In appendix I, we discuss a delta-convergent sequence useful for dimensional regularization in twistor space; and in appendix II, we discuss details of the holomorphic anomaly computation.

\section{Tree-Level Amplitudes}

We begin by reviewing the connection between tree-level amplitudes in momentum space, and the twistor-space amplitudes emerging from Witten's string theory. Rather than starting from a 


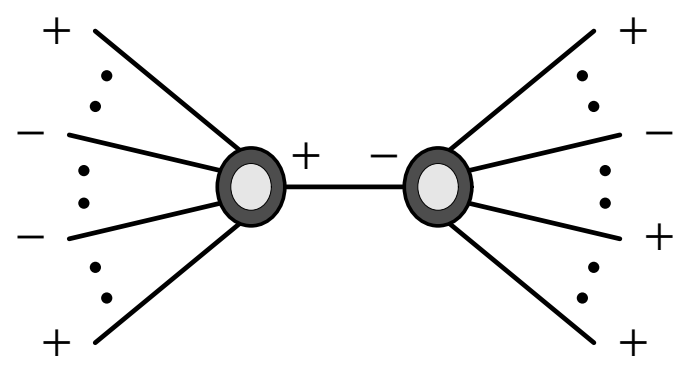

Figure 1. An example of a CSW diagram contributing to the tree amplitude with three negativehelicity gluons.

twistor-space amplitude, we begin from a momentum-space amplitude and work backwards to the twistor-space one. As our example, we take a diagram $\delta A$ contributing to an amplitude with three negative-helicity gluons or a next-to-MHV (NMHV) amplitude. This is the simplest amplitude containing more than one MHV vertex. This contribution is,

$$
\begin{gathered}
(2 \pi)^{4} \delta^{4}\left(P_{A}+P_{B}\right) \delta A\left(P_{A}, P_{B}\right)=(2 \pi)^{4} \delta^{4}\left(P_{A}\right. \\
\left.+P_{B}\right) V\left(P_{A},-P_{A}^{b}\right) \frac{i}{P_{A}^{2}} V\left(P_{B},-P_{B}^{b}\right) \\
=(2 \pi)^{4} \int d^{4} k_{1} d^{4} k_{2} \delta^{4}\left(P_{A}+k_{1}\right) V\left(P_{A}, k_{1}^{b}\right) \frac{i \delta^{4}\left(k_{1}+k_{2}\right)}{k_{1}^{2}+i \varepsilon} \\
\times \delta^{4}\left(P_{B}+k_{2}\right) V\left(P_{B}, k_{2}^{b}\right),
\end{gathered}
$$

corresponding to the diagram shown in fig. 1. In this equation, $V(P, k)$ is an MHV vertex depending on the momenta in the set $P$, and on the off-shell momentum $k$. The off-shell continuation of the MHV amplitudes to MHV vertices is defined using the projected momentum $[8,7]$,

$$
k_{a \dot{a}}^{b}=k_{a \dot{a}}-\frac{k^{2}}{2 k \cdot \eta} \eta_{a \dot{a}}
$$

where $\eta$ can be thought of as the light-cone vector of light-cone gauge. (This is equivalent to the CSW off-shell continuation.) This motivates the BST decomposition,

$$
k_{a \dot{a}}=l_{a} \tilde{l}_{\dot{a}}+z \eta_{a} \tilde{\eta}_{\dot{a}}
$$

Each momentum $p_{X i} \in P_{X}$, can be decomposed in terms of spinorial variables,

$$
p_{X i a \dot{a}}=\lambda_{X i a} \tilde{\lambda}_{X i \dot{a}}
$$

The vertices can then be thought of as functions of the $\lambda$ and $\tilde{\lambda}$. The simplification captured by the CSW rules arises because the vertices are in fact independent of the $\tilde{\lambda}_{i}$,

$$
V=V(\lambda, l)
$$


The $i \varepsilon$ in the propagator does not play a role at tree level, but will at loop level.

The twistor-space amplitude $A^{\mathrm{T}}$ is given by a 'half-Fourier transform' of the momentum-space one, that is a Fourier transform with respect to the $\tilde{\lambda}$ variables alone,

$A^{\mathrm{T}}\left(\lambda_{A}, \lambda_{B}, \mu_{A}, \mu_{B}\right)=(2 \pi)^{4} \int \prod_{i \in A} \frac{d^{2} \tilde{\lambda}_{A i \dot{a}}}{(2 \pi)^{2}} e^{i\left[\mu_{A i}, \tilde{\lambda}_{A i}\right]} \prod_{i \in B} \frac{d^{2} \tilde{\lambda}_{B i \dot{b}}}{(2 \pi)^{2}} e^{i\left[\mu_{B i}, \tilde{\lambda}_{B i}\right]} \delta^{4}\left(P_{A}+P_{B}\right) A\left(P_{A}, P_{B}\right)$.

(Note that the sign conventions on the spinor products used here and elsewhere in the twistorspace literature are different from those used conventionally in the QCD literature. We will use the notation $\left\langle\lambda_{1}, \lambda_{2}\right\rangle$ and $\left[\tilde{\lambda}_{1}, \tilde{\lambda}_{2}\right]$ in the present paper in contrast to the comma-less (and $\lambda$-less) notation $\langle 12\rangle$ and [12] standard in the QCD literature. While the two angle products agree, $\left.\left[\tilde{\lambda}_{1}, \tilde{\lambda}_{2}\right]=-[12].\right)$

Using the CSW representation (2.1), and rewriting the delta functions involving the external momenta, we obtain for the diagram of eq. (2.1),

$$
\begin{aligned}
(2 \pi)^{4} & \int \frac{d^{4} k_{1}}{(2 \pi)^{4}} \frac{d^{4} k_{2}}{(2 \pi)^{4}} \frac{i \delta^{4}\left(k_{1}+k_{2}\right)}{k_{1}^{2}+i \varepsilon} \int d^{4} x_{A}^{a \dot{a}} d^{4} x_{B}^{b \dot{b}} \int \prod_{i \in A} \frac{d^{2} \tilde{\lambda}_{A i \dot{a}}}{(2 \pi)^{2}} \prod_{i \in B} \frac{d^{2} \tilde{\lambda}_{B i \dot{b}}}{(2 \pi)^{2}} e^{i\left[\mu_{A i}, \tilde{\lambda}_{A i}\right]+i\left[\mu_{B i}, \tilde{\lambda}_{B i}\right]} \\
& \times \exp \left[i x_{A}^{a \dot{a}}\left(\sum_{i \in A} \lambda_{A i a} \tilde{\lambda}_{A i \dot{a}}+l_{1 a} \tilde{l}_{1 \dot{a}}+z_{1} \eta_{a} \tilde{\eta}_{\dot{a}}\right)\right] V\left(\lambda_{A}, l_{1}\right) \\
& \times \exp \left[i x_{B}^{b \dot{b}}\left(\sum_{i \in B} \lambda_{B i b} \tilde{\lambda}_{B i \dot{b}}+l_{1 b} \tilde{l}_{1 \dot{b}}+z_{2} \eta_{b} \tilde{\eta}_{\dot{b}}\right)\right] V\left(\lambda_{B}, l_{2}\right) .
\end{aligned}
$$

Performing the $\tilde{\lambda}_{i}$ integrals yields delta functions for the $\mu_{X i}$,

$$
\begin{aligned}
(2 \pi)^{4} & \int \frac{d^{4} k_{1}}{(2 \pi)^{4}} \frac{d^{4} k_{2}}{(2 \pi)^{4}} \frac{i \delta^{4}\left(k_{1}+k_{2}\right)}{k_{1}^{2}+i \varepsilon} \int d^{4} x_{A}^{a \dot{a}} d^{4} x_{B}^{b \dot{b}} \int d^{2} m_{1}^{\dot{a}} d^{2} m_{2}^{\dot{b}} e^{i\left[m_{1}, \tilde{l}_{1}\right]+i\left[m_{2}, \tilde{l}_{2}\right]} \\
& \times \exp \left[i x_{A}^{a \dot{a}} z_{1} \eta_{a} \tilde{\eta}_{\dot{a}}\right] V\left(\lambda_{A}, l_{1}\right) \prod_{i \in A} \delta^{2}\left(\mu_{A i}^{\dot{a}}-x_{A}^{a \dot{a}} \lambda_{A i a}\right) \delta^{2}\left(m_{1}^{\dot{a}}-x_{A}^{a \dot{a}} l_{1 a}\right) \\
& \times \exp \left[i x_{B}^{b \dot{b}} z_{2} \eta_{b} \tilde{\eta}_{\dot{b}}\right] V\left(\lambda_{B}, l_{2}\right), \prod_{i \in B} \delta^{2}\left(\mu_{B i}^{\dot{b}}-x_{B}^{b \dot{b}} \lambda_{B i b}\right) \delta^{2}\left(m_{2}^{\dot{a}}-x_{A}^{a \dot{a}} l_{2 a}\right),
\end{aligned}
$$

where we have also introduced new variables $m_{1,2}$ conjugate to $\tilde{l}_{1,2}$. Performing the $k_{2}$ integral will set $l_{2} \tilde{l}_{2}=-l_{1} \tilde{l}_{1}$ and $z_{2}=-z_{1}$; making the phase choice $l_{2}=l_{1}$ (and hence $\tilde{l}_{2}=-\tilde{l}_{1}$ ), we obtain the expression,

$$
\begin{aligned}
& \int \frac{d^{4} k}{(2 \pi)^{4}} \frac{i}{k^{2}+i \varepsilon} \int d^{4} x_{A}^{a \dot{a}} d^{4} x_{B}^{b \dot{b}} \int d^{2} m_{1}^{\dot{a}} d^{2} m_{2}^{\dot{b}} e^{i\left[\left(m_{1}-m_{2}\right), \tilde{l}\right]} \\
& \quad \times \exp \left[i\left(x_{A}-x_{B}\right)^{a \dot{a}} z \eta_{a} \tilde{\eta}_{\dot{a}}\right] V\left(\lambda_{A}, l\right) V\left(\lambda_{B}, l\right) \\
& \quad \times \prod_{i \in A} \delta^{2}\left(\mu_{A i}^{\dot{a}}-x_{A}^{a \dot{a}} \lambda_{A i a}\right) \delta^{2}\left(m_{1}^{\dot{a}}-x_{A}^{a \dot{a}} l_{a}\right) \prod_{i \in B} \delta^{2}\left(\mu_{B i}^{\dot{b}}-x_{B}^{b \dot{b}} \lambda_{B i b}\right) \delta^{2}\left(m_{2}^{\dot{a}}-x_{A}^{a \dot{a}} l_{a}\right),
\end{aligned}
$$

for the twistor-space amplitude (we have dropped the subscript ' 1 ' on $k, l$, and $\tilde{l}$ ). 
Our final task is to convert the momentum space propagator to a twistor-space one. We make use of the decomposition [12] of the loop-integral measure into the Nair measure [2] and a dispersive measure over $z$,

$$
\frac{d^{4} k}{k^{2}+i \varepsilon}=\frac{d z}{z+i \varepsilon}\left[\langle l, d l\rangle d^{2} \tilde{l}-[l, d l] d^{2} l\right] .
$$

In the twistor-space form, the integral over $z$ is just a theta function,

$$
I_{\eta}\left(x_{A}-x_{B}\right)=-i \int_{-\infty}^{\infty} \frac{d z}{2 \pi} \frac{1}{z+i \varepsilon} \exp \left[i\left(x_{A}-x_{B}\right)^{a \dot{a}} z \eta_{a} \tilde{\eta}_{\dot{a}}\right]
$$

which restricts the integral to half of the moduli space, while the $l$ and $\tilde{l}$ integrals,

$$
G_{0}\left(m_{1}, m_{2} ; l\right)=-\int \frac{1}{(2 \pi)^{3}}\left[\langle l, d l\rangle d^{2} \tilde{l}-[l, d l] d^{2} l\right] \exp \left[i\left[\left(m_{1}-m_{2}\right), \tilde{l}\right]\right]
$$

should yield the twistor-space propagator. To see this we would like to transform the measure to the CSW form. We can do this as follows. Define a real scale factor $\tau$,

$$
l=\tau l^{\prime}, \quad \tilde{l}=\tau \tilde{l}^{\prime},
$$

where

$$
l^{\prime}=(1, w), \quad \tilde{l}^{\prime}=(1, \bar{w})
$$

$(\bar{w}$ will be taken to be the complex conjugate of $w)$. Then

$$
\begin{gathered}
\langle l, d l\rangle=\tau^{2}\left\langle l^{\prime}, d l^{\prime}\right\rangle, \quad[\tilde{l}, d \tilde{l}]=\tau^{2}\left[\tilde{l}^{\prime}, d \tilde{l}^{\prime}\right], \\
2 d^{2} \tilde{l}=[d \tilde{l}, d \tilde{l}]=2 \tau d \tau\left[\tilde{l}^{\prime}, d \tilde{l}^{\prime}\right], \quad 2 d^{2} l=\langle d l, d l\rangle=2 \tau d \tau\left\langle l^{\prime}, d l^{\prime}\right\rangle,
\end{gathered}
$$

and

$$
\left[\langle l, d l\rangle d^{2} \tilde{l}-[l, d l] d^{2} l\right]=2 \tau^{3} d \tau \wedge\left\langle l^{\prime}, d l^{\prime}\right\rangle \wedge\left[\tilde{l}^{\prime}, d \tilde{l}^{\prime}\right]
$$

which is the CSW measure $\left(t_{\mathrm{CSW}}=\tau^{2}\right)$.

Now, the vertices $V$ are homogeneous functions of the spinors $\lambda_{X i a}$. Also, the two opposite ends of the propagator line correspond to gluons of opposite helicity exiting the two vertices, and so,

$$
V\left(\lambda_{A}, l\right) V\left(\lambda_{B}, l\right)=V\left(\lambda_{A}, l^{\prime}\right) V\left(\lambda_{B}, l^{\prime}\right)
$$

We can therefore ignore the vertices in evaluating $\tau$ and $\tilde{l}^{\prime}$ integrals. Write out the protopropagator (2.12) in terms of $\tau$ and $\bar{w}$,

$$
G_{0}=-\frac{1}{(2 \pi)^{3}} \int_{-\infty}^{\infty} \tau^{3} d \tau \int_{\mathbb{C}} d w d \bar{w} \exp \left[i \tau^{2}\left[m_{12}^{\prime \dot{1}} \bar{w}-m_{12}^{\prime 2}\right]\right]
$$




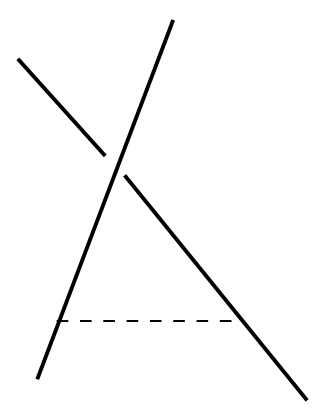

Figure 2. The twistor-space configuration contributing to an NMHV amplitude. The external points lie on a pair of lines connected by a twistor-space propagator, represented by a dashed line.

where we have also used rescaled $m_{i}^{\prime}=m_{i} / \tau$ and denoted $m_{1}^{\prime}-m_{2}^{\prime}$ by $m_{12}^{\prime}$. Factors of $\tau$ from the rescaling cancel between the $d^{2} m$ measure and the delta functions requiring that the endpoints of the propagator lie on the $x_{A, B}$ lines. We can evaluate the $\bar{w}$ integral, which yields a delta function,

$$
2 \pi \delta\left(\tau^{2} m_{12}^{\prime i}\right)=\frac{2 \pi}{\tau^{2}} \delta\left(\left(m_{1}^{\prime}-m_{2}^{\prime}\right)^{\dot{1}}\right),
$$

where the equality follows from the factors of $\tau$ in the integration measure in eq. (2.18). In order to do the $\tau$ integral, we must introduce an $i \varepsilon$ prescription for $m_{12}^{\prime 2} \rightarrow m_{12}^{\prime 2}-i \varepsilon$. If we now do the $\tau$ integral, we obtain

$$
\begin{aligned}
& \frac{i}{2 \pi} \frac{\delta\left(m_{12}^{\prime i}\right)}{m_{12}^{\prime 2}-i \varepsilon} \int_{-\infty}^{\infty} \frac{d w}{2 \pi} \\
& =\frac{i}{2 \pi} \frac{\delta\left(m_{12}^{\prime i}\right)}{m_{12}^{\prime 2}-i \varepsilon} \int_{-\infty}^{\infty} \frac{d w_{1}}{2 \pi} \frac{d w_{2}}{2 \pi} 2 \pi \delta\left(w_{1}-w_{2}\right) .
\end{aligned}
$$

We cannot do the $w$ integral, because the vertices do depend on this variable. Indeed, these remaining integrals represent the $l$ integrations over the endpoints of the propagator, and should be thought of as part of the moduli space of curves for this amplitude. The delta function $\delta\left(w_{1}-w_{2}\right)$ on the second line should be considered part of the propagator.

Define a propagator without the $w$ integral,

$$
G\left(m_{12}^{\prime}\right) \equiv \frac{i}{2 \pi} \frac{\delta\left(m_{12}^{\prime i}\right)}{m_{12}^{\prime 2}-i \varepsilon}
$$

Alternatively, averaging over both order of evaluation of the $\tau$ and $\bar{w}$ integrals in eq. (2.18), we obtain

$$
G_{2}\left(m_{12}^{\prime}\right)=\frac{i}{4 \pi}\left[\frac{\delta\left(m_{12}^{\prime i}\right)}{m_{12}^{\prime 2}-i \varepsilon}+\frac{\delta\left(m_{12}^{\prime 2}\right)}{m_{12}^{\prime i}-i \varepsilon}\right] .
$$


(In the second order of evaluation we have used a principal-value prescription.) At tree level both eq. (2.21) and eq. (2.22) lead to the same answer. Inserting the former into eq. (2.9), we obtain the final expression for the tree-level diagram,

$$
\begin{aligned}
\int d^{4} & x_{A}^{a \dot{a}} d^{4} x_{B}^{b \dot{b}} \int \frac{d l^{\prime}}{2 \pi} d^{2} m_{1}^{\prime \dot{a}} d^{2} m_{2}^{\prime \dot{b}} \\
& \times V\left(\lambda_{A}, l^{\prime}\right) V\left(\lambda_{B}, l^{\prime}\right) I_{\eta}\left(x_{A}-x_{B}\right) G\left(m_{12}^{\prime}\right) \\
& \times \prod_{i \in A} \delta^{2}\left(\mu_{A i}^{\dot{a}}-x_{A}^{a \dot{a}} \lambda_{A i a}\right) \delta^{2}\left(m_{1}^{\prime \dot{a}}-x_{A}^{a \dot{a}} l_{a}^{\prime}\right) \prod_{i \in B} \delta^{2}\left(\mu_{B i}^{\dot{b}}-x_{B}^{b \dot{b}} \lambda_{B i b}\right) \delta^{2}\left(m_{2}^{\prime \dot{a}}-x_{A}^{a \dot{a}} l_{a}^{\prime}\right) .
\end{aligned}
$$

This result is equivalent to the integrals considered in sect. 6 of ref. [5]. It expresses this diagram's contribution to the twistor-space amplitude as a function supported on a pair of lines connected by a propagator, illustrated in fig. 2 .

\section{Hunting Infrared Divergences}

From the results of ref. [12] we may expect, as also noted in ref. [18], that the simplest loop amplitude in twistor space (an MHV amplitude) would be obtained by connecting the two lines in the tree-level amplitude of sect. 2 by another propagator, so as to obtain a genus-one configuration. This will indeed turn out to be the case. To reach such a representation, however, we must first confront the infrared singularities in the amplitude.

These infrared singularities are conventionally regulated dimensionally in perturbative gauge theories. In momentum space, they arise from configurations where the loop momentum is nearly on shell and either soft or collinear with an external momentum (or both). When computing in $D=4-2 \epsilon$ dimensions, with $\epsilon<0$, the singularities manifest themselves in the appearance of poles in $\epsilon$. In gauge theories, the leading singularities are a factor of $1 / \epsilon^{2}$ per loop order.

The one-loop amplitude for the MHV amplitude in $\mathcal{N}=4$ supersymmetric gauge theory was computed a decade ago, by Dixon, Dunbar, and two of the authors [13]. The leading-color or planar contributions* have the form of a sum over color permutations, with each term in the sum equal to,

$$
A_{n}^{1 \text {-loop }}=c_{\Gamma} A_{n}^{\text {tree }} V_{n}^{g}
$$

\footnotetext{
* The subleading-color terms can be obtained algebraically from the leading-color ones as discussed in sect. 7 of ref. [13].
} 
where

$$
\begin{aligned}
c_{\Gamma} & =\frac{1}{(4 \pi)^{2-\epsilon}} \frac{\Gamma(1+\epsilon) \Gamma^{2}(1-\epsilon)}{\Gamma(1-2 \epsilon)} \\
V_{2 m+1}^{g} & =\left(\mu^{2}\right)^{\epsilon} \sum_{r=2}^{m-1} \sum_{i=1}^{n} F_{n: r ; i}^{2 \mathrm{~m} e}+\sum_{i=1}^{n} F_{n: i}^{1 \mathrm{~m}}, \\
V_{2 m}^{g} & =\left(\mu^{2}\right)^{\epsilon} \sum_{r=2}^{m-2} \sum_{i=1}^{n} F_{n: r ; i}^{2 \mathrm{~m} e}+\sum_{i=1}^{n} F_{n: i}^{1 \mathrm{~m}}+\sum_{i=1}^{n / 2} F_{n: m-1 ; i}^{2 \mathrm{~m} e} .
\end{aligned}
$$

The integral functions $F^{2 \mathrm{~m} e}$ and $F^{1 \mathrm{~m}}$ are given in terms of

$$
\begin{aligned}
F\left(s, t, P^{2}, Q^{2}\right)= & -\frac{1}{\epsilon^{2}}\left[(-s)^{-\epsilon}+(-t)^{-\epsilon}-\left(-P^{2}\right)^{-\epsilon}-\left(-Q^{2}\right)^{-\epsilon}\right] \\
& +\operatorname{Li}_{2}\left(1-\frac{P^{2}}{s}\right)+\operatorname{Li}_{2}\left(1-\frac{P^{2}}{t}\right)+\operatorname{Li}_{2}\left(1-\frac{Q^{2}}{s}\right)+\operatorname{Li}_{2}\left(1-\frac{Q^{2}}{t}\right) \\
& -\operatorname{Li}_{2}\left(1-\frac{P^{2} Q^{2}}{s t}\right)+\frac{1}{2} \ln ^{2}\left(\frac{s}{t}\right),
\end{aligned}
$$

as follows,

$$
\begin{aligned}
F_{n: i}^{1 \mathrm{~m}} & =F\left(s_{(i-3)(i-2)}, s_{(i-2)(i-1)}, s_{i \cdots(i+n-4)}, 0\right), \\
F_{n: r ; i}^{2 \mathrm{~m} e} & =F\left(s_{(i-1) \cdots(i+r-1)}, s_{i \cdots(i+r)}, s_{i \cdots(i+r-1)}, s_{(i+r+1) \cdots(i-2)}\right),
\end{aligned}
$$

where $s_{j_{1} \cdots j_{2}}=K_{j_{1} \cdots j_{2}}^{2}=\left(k_{j_{1}}+\cdots+k_{j_{2}}\right)^{2}$ and all indices are understood cyclicly $\bmod n$. ( $F$ has a smooth limit as $P^{2}$ or $Q^{2} \rightarrow 0$.) These formulæ make it appear as though all diagrams will contribute infrared-divergent terms.

Appearances can be deceiving, however, and in this case, they are. Each diagram built out of MHV vertices corresponds closely to a cut in a specific channel in a unitarity-based calculation [19,20], which uses on-shell amplitudes on both sides of a cut. This close correspondence allows us to show that most MHV diagrams are in fact free of infrared divergences, which are present only in a subset of diagrams. Indeed, if we examine a generic cut of the amplitude, say in the $s_{c_{1} \cdots c_{2}}$ channel $\left(\left|c_{1}-c_{2}\right|>1\right)$, we find,

$$
\begin{aligned}
& 2 \pi i c_{\Gamma} A^{\text {tree }} \\
& \quad \times \ln \left(\frac{\left(s_{\left(c_{1}-1\right) \cdots\left(c_{2}-1\right)} s_{c_{1} \cdots c_{2}}-s_{c_{1} \cdots\left(c_{2}-1\right)} s_{\left(c_{1}-1\right) \cdots c_{2}}\right)\left(s_{c_{1} \cdots c_{2}} s_{\left(c_{1}+1\right) \cdots\left(c_{2}+1\right)}-s_{c_{1} \cdots\left(c_{2}+1\right)} s_{\left(c_{1}+1\right) \cdots c_{2}}\right)}{\left(s_{c_{1} \cdots\left(c_{2}-1\right)} s_{\left(c_{1}+1\right) \cdots c_{2}}-s_{c_{1} \cdots c_{2}} s_{\left(c_{1}+1\right) \cdots\left(c_{2}-1\right)}\right)\left(s_{\left(c_{1}-1\right) \cdots c_{2}} s_{c_{1} \cdots\left(c_{2}+1\right)}-s_{c_{1} \cdots c_{2}} s_{\left(c_{1}-1\right) \cdots\left(c_{2}+1\right)}\right)}\right),
\end{aligned}
$$

a finite result. But the infrared-divergent contributions to the amplitude arise from infrareddivergent contributions to the cuts - one power of $1 / \epsilon$ arises from the cut integral, and the other from the dispersion integral. As shown by Brandhuber, Spence, and Travaglini, however, the cut of the twistor-rules diagram built out of the two MHV vertices $V\left(\ell_{1}, c_{1}, \ldots, c_{2}, \ell_{2}\right)$ and $V\left(-\ell_{2}, c_{2}+1, \ldots, c_{1}-1,-\ell_{1}\right)$ is exactly the same as the above cut. This in turn implies that the twistor-rules diagram does not have infrared divergences either. 
Indeed, we know on general grounds [21] that the infrared divergences of a one-loop diagram in the $\mathcal{N}=4$ theory are of the form,

$$
V_{n, \mathrm{IR}}^{g}=-\frac{\left(\mu^{2}\right)^{\epsilon}}{\epsilon^{2}} \sum_{i=1}^{n}\left(-s_{i(i+1)}\right)^{-\epsilon}
$$

involving only nearest-neighbor two-particle invariants. In the MHV amplitude, these arise from twistor-rules diagrams where one of the vertices is a four-point vertex.

We can understand this result as well [22] by looking at the cuts of diagrams. Infrared divergences in the cuts can arise only from regions where the phase-space integral diverges as $\epsilon \rightarrow 0$. For generic values of the external momenta, singularities in multiparticle invariants are at isolated points, and hence of insufficient dimensionality to induce such a divergence. Only two-particle invariants will produce such a divergence. However, in general the only two-particle singularities involving the cut loop momentum are collinear singularities, where that momentum becomes collinear to a neighboring external momentum. These are characteristically $1 / \sqrt{s_{i j}}$ singularities, which are integrable even as $\epsilon \rightarrow 0$. (Here, it is crucial that each side of the cut is an amplitude, and not merely a Feynman tree diagram; in most gauges, this statement is not true diagram by diagram.)

In the particular case of the MHV loop amplitude, in the channel we are examining, there are four candidate regions, corresponding to the inverse spinor products

$$
\frac{1}{\left\langle\ell_{1}, c_{1}\right\rangle}, \quad \frac{1}{\left\langle\ell_{1},\left(c_{1}-1\right)\right\rangle}, \quad \frac{1}{\left\langle\ell_{2},\left(c_{2}+1\right)\right\rangle}, \quad \text { and } \frac{1}{\left\langle\ell_{2}, c_{2}\right\rangle}
$$

where $\ell_{i}$ are the cut momenta. Each of these expressions is singular in collinear regions $\left(\ell_{1} \| k_{c_{1}}\right.$, etc.), but that singularity only goes as $1 / \theta_{\ell c_{1}}$. The measure is $d(\cos \theta) \sim \theta d \theta$, so the expressions are integrable, and give rise to no infrared singularity.

The exception is of course the cut where the amplitude on one side is a four-point amplitude. In this case, when one of the cut loop momenta becomes collinear to the neighboring external momentum, momentum conservation forces the other cut loop momentum to become collinear to its neighboring external momentum, and both $1 / \sqrt{s_{i j}}$ singularities overlap to produce a $1 / s_{i j}$-type singularity or a $d \theta / \theta$ integral, which does give rise to a $1 / \epsilon$ pole when regulated dimensionally. (This singularity in the tree amplitude should be characterized as a forward-scattering singularity rather than as a collinear singularity.)

The twistor-rules diagrams contributing to the one-loop amplitude thus fall into two classes: (a) diagrams containing a four-point vertex, which are infrared divergent, and require an implementation of dimensional regularization, and (b) all other diagrams, which are infrared-finite, and 


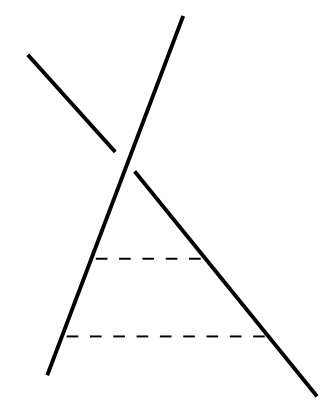

Figure 3. The twistor-space configuration contributing to an MHV amplitude. The external points lie on a pair of lines connected by two twistor-space propagators.

hence can be treated in four dimensions. (Note that diagrams with a three-point vertex have vanishing cut and so can be ignored.) We consider the twistor-space form of the latter diagrams in the next section.

\section{Infrared-Finite Contributions to One-Loop Amplitudes}

We can obtain the expression for a single one-loop twistor-rules diagram from the tree-level one (2.1) by adding one additional propagator, along with appropriate delta functions to produce a genus-one configuration,

$$
\begin{gathered}
\int d^{4} k_{A 1} d^{4} k_{B 1} d^{4} k_{A 2} d^{4} k_{B 2} \delta^{4}\left(k_{A 1}+P_{A}+k_{A 2}\right) V\left(k_{A 1}^{b}, P_{A}, k_{A 2}^{b}\right) \frac{i \delta^{4}\left(k_{A 1}+k_{B 1}\right)}{k_{A 1}^{2}+i \varepsilon} \frac{i \delta^{4}\left(k_{A 2}+k_{B 2}\right)}{k_{A 2}^{2}+i \varepsilon} \\
\times \delta^{4}\left(k_{B 1}+P_{B}+k_{B 2}\right) V\left(k_{B 2}^{b}, P_{B}, k_{B 1}^{b}\right) .
\end{gathered}
$$

In this section, we restrict attention to diagrams where both vertices have more than four legs (including the sewn legs). As we have seen above, these integrals are infrared-convergent and can be treated in four dimensions.

Following similar manipulations as in sect. 2, we find the following expression for the contri- 
bution of a twistor-rules diagram to the one-loop twistor-space amplitude,

$$
\begin{aligned}
\int d^{4} & x_{A}^{a \dot{a}} d^{4} x_{B}^{b \dot{b}} \int \frac{d l_{1}^{\prime}}{2 \pi} \frac{d l_{2}^{\prime}}{2 \pi} \int d^{2} m_{A 1}^{\prime \dot{a}} d^{2} m_{A 2}^{\prime \dot{a}} \int d^{2} m_{B 1}^{\prime \dot{b}} d^{2} m_{B 2}^{\prime \dot{b}} \\
& \times V\left(l_{1}^{\prime}, \lambda_{A}, l_{2}^{\prime}\right) V\left(l_{2}^{\prime}, \lambda_{B}, l_{1}^{\prime}\right) I_{\eta}\left(x_{A}-x_{B}\right) G_{2}\left(m_{A 1, B 1}^{\prime}\right) G_{2}\left(m_{A 2, B 2}^{\prime}\right) \\
& \times \prod_{i \in A} \delta^{2}\left(\mu_{A i}^{\dot{a}}-x_{A}^{a \dot{a}} \lambda_{A i a}\right) \delta^{2}\left(m_{A 1}^{\prime \dot{a}}-x_{A}^{a \dot{a}} l_{1 a}^{\prime}\right) \delta^{2}\left(m_{B 1}^{\prime \dot{b}}-x_{B}^{b \dot{b}} l_{1 b}^{\prime}\right) \\
& \times \prod_{i \in B} \delta^{2}\left(\mu_{B i}^{\dot{b}}-x_{B}^{b \dot{b}} \lambda_{B i b}\right) \delta^{2}\left(m_{A 2}^{\prime \dot{a}}-x_{A}^{a \dot{a}} l_{2 a}^{\prime}\right) \delta^{2}\left(m_{B 2}^{\prime \dot{b}}-x_{B}^{b \dot{b}} l_{2 b}^{\prime}\right) .
\end{aligned}
$$

This expression gives the complete contribution for diagrams where the two external gluons with negative helicity are attached to the same vertex, because only gluons can circulate in the loop. The use of $G_{2}$ reflects subtleties with the $i \epsilon$ prescription. For other diagrams, we must include the contributions of the full $\mathcal{N}=4$ multiplet by sprinkling fermionic integrals and delta functions into this equation. This result expresses the contribution as a function supported on a pair of lines connected by two propagators into a genus-one topology, illustrated in fig. 3. This is a leading-color contribution, as dictated by the ordering of points inside the two vertex functions $V$.

\section{Infrared-Divergent One-Loop Diagrams}

When the twistor-rules diagram does contain a four-point vertex, it will be infrared divergent. As we have discussed in sect. 3 , these divergences arise from regions of the $l_{i}$ integration where $l_{1}$ becomes collinear to $\lambda_{1}$. Momentum conservation then forces $l_{2}$ to become collinear to $\lambda_{2}$. While it is not clear how to interpret the usual momentum-space regulator $\left(d^{4} p \rightarrow d^{D} p\right)$ in twistor space, it isn't necessary to have such an interpretation.

One way to regulate the divergence is to smear out the energy-momentum conserving delta function in eq. (4.1), replacing

$$
\delta^{4}\left(k_{A 1}+P_{A}+k_{A 2}\right) \rightarrow \Delta_{\xi}^{4}\left(k_{A 1}+P_{A}+k_{A 2}\right),
$$

where $\Delta_{\xi}$ is a delta-convergent sequence, for example $\Delta_{\xi}(x)=\xi /\left(\xi^{2}+x^{2}\right)$. In this case, one could perform all the conversions to twistor space, and study the limit later. The infrared divergences manifest themselves as logarithmic divergences in the $\xi \rightarrow 0$ limit.

We will take a different approach, and simply write down a regulator in twistor space that is designed to reproduce the usual answer from momentum-space dimensional regularization. Suppose that it is set $A$ that is on the four-point vertex, and thus contains two external legs; if these two are labeled 1 and 2, then the diagram will result in a contribution

$$
\sim\left(-s_{12}\right)^{-\epsilon} f\left(s_{12}, \cdots ; \epsilon\right),
$$


where the leading behavior of $f$ is $-1 / \epsilon^{2}$. We know that we need to regulate the behavior of the $l_{1}$ integral near the collinear region, softening the pole there ${ }^{\dagger}$. We can do so by introducing a factor of $\left\langle l_{1}, \lambda_{1}\right\rangle^{-\epsilon}$, with $\epsilon<0$, into the integrand. For symmetry, we also introduce a similar factor for $l_{2}$. If we then make the resulting factor homogeneous in the $l_{i}$, we find,

$$
R_{\epsilon}\left(l_{1}, l_{2}, \lambda_{1}, \lambda_{2}\right)=\left(\frac{\left\langle l_{1}, \lambda_{1}\right\rangle\left\langle l_{2}, \lambda_{2}\right\rangle}{\left\langle l_{1}, l_{2}\right\rangle}\right)^{-\epsilon}
$$

for our regulating factor.

We are not quite done. The above factor will result in a pole contribution proportional to $\left\langle\lambda_{1}, \lambda_{2}\right\rangle^{-\epsilon}$, which is not exactly of the desired form; we are missing a factor of $\left[\tilde{\lambda}_{1}, \tilde{\lambda}_{2}\right]^{-\epsilon}$. We can determine the required factor in the twistor-space integral by multiplying the original momentumspace amplitude by this factor, and then performing the Fourier transforms with respect to the $\tilde{\lambda}$ variables as in sect. 2. The computation of the Fourier transform is given in appendix I. The result there shows that in order to introduce the desired factor into the result, we should modify the integrand, replacing the delta functions containing $m_{A 1}$ and $m_{A 2}$ by a delta-convergent sequence,

$$
\delta^{2}\left(m_{A 1}^{\dot{a}}-x_{A}^{a \dot{a}} l_{a}^{\prime}\right) \delta^{2}\left(m_{A 2}^{\dot{a}}-x_{A}^{a \dot{a}} l_{a}^{\prime}\right) \rightarrow \frac{\epsilon \Gamma(2-\epsilon)}{(2 \pi)^{3} \Gamma(1+\epsilon)}\left[m_{A 1}^{\prime}-x_{A}^{a} l_{1 a}^{\prime}, m_{A 2}^{\prime}-x_{A}^{a} l_{2 a}^{\prime}\right]^{\epsilon-2} .
$$

The resulting expression for this loop diagram in twistor space is thus,

$$
\begin{aligned}
& \frac{\epsilon \Gamma(2-\epsilon)}{(2 \pi)^{3} \Gamma(1+\epsilon)} \int d^{4} x_{A}^{a \dot{a}} d^{4} x_{B}^{b \dot{b}} \int \frac{d l_{1}^{\prime}}{2 \pi} \frac{d l_{2}^{\prime}}{2 \pi} \int d^{2} m_{A 1}^{\prime \dot{a}} d^{2} m_{A 2}^{\dot{a}} \int d^{2} m_{B 1}^{\prime \dot{b}} d^{2} m_{B 2}^{\prime \dot{b}} \\
& \times R_{\epsilon}\left(l_{1}, l_{2}, \lambda_{1}, \lambda_{2}\right) V\left(l_{1}^{\prime}, \lambda_{A}, l_{2}^{\prime}\right) V\left(l_{2}^{\prime}, \lambda_{B}, l_{1}^{\prime}\right) I_{\eta}\left(x_{A}-x_{B}\right) G\left(m_{A 1, B 1}^{\prime}\right) G\left(m_{A 2, B 2}^{\prime}\right) \\
& \times\left[m_{A 1}^{\prime}-x_{A}^{a} l_{1 a}^{\prime}, m_{A 2}^{\prime}-x_{A}^{a} l_{2 a}^{\prime}\right]^{\epsilon-2} \prod_{i \in A} \delta^{2}\left(\mu_{A i}^{\dot{a}}-x_{A}^{a \dot{a}} \lambda_{A i a}\right) \delta^{2}\left(m_{B 1}^{\prime \dot{b}}-x_{B}^{b \dot{b}} l_{1 b}^{\prime}\right) \\
& \times \prod_{i \in B} \delta^{2}\left(\mu_{B i}^{\dot{b}}-x_{B}^{b \dot{b}} \lambda_{B i b}\right) \delta^{2}\left(m_{B 2}^{\prime \dot{b}}-x_{B}^{b \dot{b}} l_{2 b}^{\prime}\right),
\end{aligned}
$$

up to over-all $\epsilon$-dependent factors with a smooth $\epsilon \rightarrow 0$ limit. As noted in the previous section, it is straightforward to include fermion and scalar contributions.

\section{Dentistry with Differential Operators}

Amplitudes in twistor space have simple properties. At tree level, they are supported, as it turns out, on loci of intersecting lines. This means the amplitudes contain delta functions, so that

$\dagger$ Because we have smeared out the energy-momentum conserving delta function by representing it as an exponential integral over $x$, the $l_{1,2}$ integrals are not actually divergent. However, if they are left unregulated, the $x$ integral will turn out to be divergent. Although it may be possible to regulate the $x$ integral instead, it seems more straightforward to regulate the $l_{i}$ integrals. 
the twistor-space amplitudes satisfy certain algebraic relations. In particular, certain polynomials multiplying the amplitudes will yield a function which vanishes everywhere. Unfortunately, the coefficients of the delta functions are quite difficult to calculate directly.

As Witten pointed out in his original paper [1], however, we do not need the twistor-space amplitudes in order to establish the structure of the delta functions they contain. In momentum space, the Fourier transform turns the polynomials into differential operators (polynomial in the $\lambda_{i}$, and derivatives with respect to the $\tilde{\lambda}_{i}$ ), which will annihilate the amplitude. One particularly useful building block for these differential operators is the line annihilation operator, expressing the condition that three points in twistor space lie on a common 'line' or $\mathbb{C P}^{1}$. If the coordinates of the three points are $Z_{i=1,2,3}^{I}=\left(\lambda_{i}^{a}, \mu_{i}^{\dot{a}}\right)$, the appropriate condition is

$$
\epsilon_{I J K L} Z_{1}^{I} Z_{2}^{J} Z_{3}^{K}=0
$$

for all choices of $L$. Choosing $L=\dot{a}$, and translating this equation back to momentum space using the identification $\mu^{\dot{a}} \leftrightarrow-i \partial / \partial \lambda_{\dot{a}}$, we obtain the operator,

$$
F_{123}=\left\langle\lambda_{1}, \lambda_{2}\right\rangle \frac{\partial}{\partial \tilde{\lambda}_{3}}+\left\langle\lambda_{2}, \lambda_{3}\right\rangle \frac{\partial}{\partial \tilde{\lambda}_{1}}+\left\langle\lambda_{3}, \lambda_{1}\right\rangle \frac{\partial}{\partial \tilde{\lambda}_{2}}
$$

The tree-level MHV amplitude, for example, is annihilated by $F_{i j k}$, because it is independent of the $\tilde{\lambda}_{i}$ (and any possible delta functions vanish for generic momenta). However, as noted by Cachazo, Svrček, and Witten [17], not all such operators (nor products of them corresponding to configurations with all points on two intersecting lines [fig. 1(b) of that paper]) annihilate the finite parts of the one-loop amplitude. This is why they originally interpreted parts of the amplitude as having one of the points wandering off the line into the plane defined by their intersection, and also why they interpreted the amplitude as having derivative-of-delta function support in that plane.

If we interpret $\tilde{\lambda}_{i}$ as the complex conjugate of $\lambda_{i}$, as we must for real Minkowski momenta, then we must take into account [18], the fact that $\partial_{\bar{z}}(1 / z) \neq 0$; for spinor products,

$$
\eta^{\dot{a}} \frac{\partial}{\partial \bar{\lambda}^{\dot{a}}} \frac{1}{\langle\lambda, \chi\rangle}=2 \pi[\bar{\eta}, \bar{\chi}] \delta(\langle\lambda, \chi\rangle) \delta([\bar{\lambda}, \bar{\chi}])
$$

where $\bar{\lambda}_{i}$ denotes the complex conjugate of $\lambda_{i}$. In integrating over the cut loop momenta to compute a one-loop amplitude, we will necessarily encounter configurations where the delta function does not vanish. These give rise to the 'holomorphic anomaly' contribution, which we now proceed to compute explicitly for the MHV amplitude.

For notational clarity, let us take $c_{1}=1$, so that we are considering the cut in the $s_{1 \cdots c_{2}}$ 
channel,

$$
\begin{aligned}
& \int \frac{d^{4} \ell_{1} \delta^{(+)}\left(\ell_{1}^{2}\right)}{(2 \pi)^{3}} \frac{d^{4} \ell_{2} \delta^{(+)}\left(\ell_{2}^{2}\right)}{(2 \pi)^{3}}(2 \pi)^{4} \delta^{4}\left(K_{1 \cdots c_{2}}-\ell_{1}-\ell_{2}\right) \\
& \quad \times A^{\text {tree }}\left(\left(-\ell_{1}\right)^{+}, 1^{+}, \ldots, m_{1}^{-}, \ldots, m_{2}^{-}, \ldots, c_{2}^{+},\left(-\ell_{2}\right)^{+}\right) A^{\text {tree }}\left(\ell_{2}^{-},\left(c_{2}+1\right)^{+}, \ldots, n^{+}, \ell_{1}^{-}\right) \\
& =C \int \frac{d^{4} \ell_{1} \delta^{(+)}\left(\ell_{1}^{2}\right)}{(2 \pi)^{3}} \frac{d^{4} \ell_{2} \delta^{(+)}\left(\ell_{2}^{2}\right)}{(2 \pi)^{3}}(2 \pi)^{4} \delta^{4}\left(K_{1 \cdots c_{2}}-\ell_{1}-\ell_{2}\right) \frac{\left\langle\ell_{1}, \ell_{2}\right\rangle^{2}}{\left\langle\ell_{1}, \lambda_{1}\right\rangle\left\langle\lambda_{c_{2}}, \ell_{2}\right\rangle\left\langle\ell_{2}, \lambda_{c_{2}+1}\right\rangle\left\langle\lambda_{n}, \ell_{1}\right\rangle},
\end{aligned}
$$

where $\delta^{(+)}\left(\ell^{2}\right)=\Theta\left(\ell^{0}\right) \delta\left(\ell^{2}\right)$, and with

$$
C=-i\left\langle\lambda_{c_{2}}, \lambda_{c_{2}+1}\right\rangle\left\langle\lambda_{n}, \lambda_{1}\right\rangle A^{\text {tree }}\left(1^{+}, \ldots, m_{1}^{-}, \ldots, m_{2}^{-}, \ldots, n^{+}\right) .
$$

Consider the result of applying $\eta^{\dot{a}} F_{123 \dot{a}}$ to this cut. Only the derivative $\partial / \partial \bar{\lambda}_{1}$ can lead to a delta function term, because only $\lambda_{1}$ amongst $\lambda_{1,2,3}$ appears in an inverse spinor product with one of the cut loop momenta $\ell_{i}$. Using eq. (6.3), this delta function or 'anomaly' term is,

$$
\begin{gathered}
\eta^{\dot{a}} \Delta_{123 \dot{a}}=2 \pi C\left\langle\lambda_{2}, \lambda_{3}\right\rangle \int \frac{d^{4} \ell_{1} \delta^{(+)}\left(\ell_{1}^{2}\right)}{(2 \pi)^{3}} \frac{d^{4} \ell_{2} \delta^{(+)}\left(\ell_{2}^{2}\right)}{(2 \pi)^{3}}(2 \pi)^{4} \delta^{4}\left(K_{1 \cdots c_{2}}-\ell_{1}-\ell_{2}\right) \\
\times \frac{\left[\bar{\eta}, \bar{\ell}_{1}\right] \delta\left(\left\langle\lambda_{1}, \ell_{1}\right\rangle\right) \delta\left(\left[\bar{\lambda}_{1}, \bar{\ell}_{1}\right]\right)\left\langle\ell_{1}, \ell_{2}\right\rangle^{2}}{\left\langle\lambda_{c_{2}}, \ell_{2}\right\rangle\left\langle\ell_{2}, \lambda_{c_{2}}+1\right\rangle\left\langle\lambda_{n}, \ell_{1}\right\rangle}
\end{gathered}
$$

The cut integral, which has eight integration variables, also contains eight delta functions. As we shall show in Appendix II, these are all independent, and determine the integration variables completely. The delta functions require that $\left\langle\ell_{1}, \lambda_{1}\right\rangle$ vanish, or in other words that $\ell_{1}$ be collinear to $k_{1}$,

$$
\ell_{1}=a k_{1}
$$

The constant a may be determined from the requirement that $\ell_{2}$ be massless,

$$
a=\frac{s_{1 \cdots c_{2}}}{2 k_{1} \cdot K_{1 \cdots c_{2}}}
$$

so that

$$
\ell_{2}=K_{1 \cdots c_{2}}-\frac{s_{1 \cdots c_{2}}}{2 k_{1} \cdot K_{1 \cdots c_{2}}} k_{1} \equiv K^{\natural} \quad\left({ }^{\prime} K \text {-natural') } .\right.
$$

Because all the integration variables are determined, the result is obtained by inserting the delta-function constraints (6.7) and (6.9) into the product of tree amplitudes and multiplying by a jacobian factor,

$$
2 \pi C\left\langle\lambda_{2}, \lambda_{3}\right\rangle \mathcal{J} \frac{s_{1 \cdots c_{2}}}{2 k_{1} \cdot K_{1 \cdots c_{2}}} \frac{\left[\bar{\eta}, \bar{\lambda}_{1}\right]\left\langle\lambda_{1}, K^{\natural}\right\rangle^{2}}{\left\langle\lambda_{c_{2}}, K^{\natural}\right\rangle\left\langle K^{\natural}, \lambda_{c_{2}+1}\right\rangle\left\langle\lambda_{n}, \lambda_{1}\right\rangle} .
$$

The jacobian factor $\mathcal{J}$ arises from the integration over the delta functions and is computed in appendix II. The result is

$$
\mathcal{J}=\frac{c_{\Gamma}}{2 k_{1} \cdot K_{1 \cdots c_{2}}} .
$$


where the $\mathcal{O}(\epsilon)$ terms implicit in $c_{\Gamma}$ are irrelevant because we are considering infrared finite amplitudes here.

Multiplying and dividing eq. (6.10) by $\left[K^{\natural}, \bar{\lambda}_{1}\right]^{2}$, we can rewrite it without the explicit appearance of $K^{\natural}$,

$$
\eta^{\dot{a}} \Delta_{123 \dot{a}}=-2 \pi C\left\langle\lambda_{2}, \lambda_{3}\right\rangle \frac{\left[\bar{\eta}, \bar{\lambda}_{1}\right] s_{1 \cdots c_{2}}}{\left\langle\lambda_{c_{2}}{ }^{-}\left|\not K_{1 \cdots c_{2}}\right| \bar{\lambda}_{1}^{-}\right\rangle\left\langle\lambda_{c_{2}+1}{ }^{-}\left|\not K_{1 \cdots c_{2}}\right| \bar{\lambda}_{1}{ }^{-}\right\rangle\left\langle\lambda_{n}, \lambda_{1}\right\rangle},
$$

where

$$
\left\langle\lambda_{1}{ }^{-}|\not K| \bar{\lambda}_{2}{ }^{-}\right\rangle=\lambda_{1}^{a} K_{a \dot{a}} \bar{\lambda}_{2}{ }^{\dot{a}}
$$

Although we have focused on the computation of the anomaly for the cut MHV amplitude, the computation for other amplitudes is similar. The jacobian is universal; and the spinorial delta functions depend only on the potential collinear singularities of the cut momenta with an external momentum. That in turn is governed by universal splitting amplitudes.

We can now define a modified line operator,

$$
\eta^{\dot{a}} F_{123 \dot{a}}^{\prime}=\eta^{\dot{a}}\left(F_{123 \dot{a}}-\Delta_{123 \dot{a}}\right) .
$$

The twistor-space picture expected from the BST computation, and made explicit in sect. 4, is that the diagram corresponding to the cut considered here will be supported on configurations where points $1, \ldots, c_{2}$ lie on one line, and points $\left(c_{2}+1\right), \ldots, n$ lie on another line. Taking into account the 'holomorphic anomaly' means that this should be reflected not in $F_{123} A_{\text {cut }}=0$, but rather in

$$
F_{123}^{\prime} A_{\text {cut }}=0
$$

Let us now check this equation. Using the spinor factorization [23]

$$
(P+p)^{2}(P+q)^{2}-P^{2} Q^{2}=\left\langle p^{-}|P| q^{-}\right\rangle\left\langle q^{-}|P| p^{-}\right\rangle,
$$

we can rewrite the cut (3.5) in a more convenient form (setting $c_{1}=1$ ),

$$
\begin{aligned}
D= & \pi i c_{\Gamma} A^{\text {tree }} \\
& \times \ln \left(\frac{\left\langle\lambda_{n}{ }^{-}\left|K_{1 \cdots c_{2}}\right| \bar{\lambda}_{c_{2}}{ }^{-}\right\rangle\left\langle\lambda_{c_{2}}{ }^{-}\left|\not K_{1 \cdots c_{2}}\right| \bar{\lambda}_{n}{ }^{-}\right\rangle\left\langle\lambda_{1}{ }^{-}\left|K_{1 \cdots c_{2}}\right| \bar{\lambda}_{c_{2}+1}{ }^{-}\right\rangle\left\langle\lambda_{c_{2}+1}{ }^{-}\left|K_{1 \cdots c_{2}}\right| \bar{\lambda}_{1}{ }^{-}\right\rangle}{\left\langle\lambda_{1}{ }^{-}\left|K_{1 \cdots c_{2}}\right| \bar{\lambda}_{c_{2}}{ }^{-}\right\rangle\left\langle\lambda_{c_{2}}{ }^{-}\left|\not K_{1 \cdots c_{2}}\right| \bar{\lambda}_{1}{ }^{-}\right\rangle\left\langle\lambda_{n}{ }^{-}\left|\not K_{1 \cdots c_{2}}\right| \bar{\lambda}_{c_{2}+1}{ }^{-}\right\rangle\left\langle\lambda_{c_{2}+1}{ }^{-}\left|K_{1 \cdots c_{2}}\right| \bar{\lambda}_{n}{ }^{-}\right\rangle}\right) .
\end{aligned}
$$

Now, $F^{\prime}$ is a linear operator, so we can consider its action on the cut as the sum of its actions on different factors inside the cut. In particular, $F_{123}$ (and hence $F_{123}^{\prime}$ ) annihilates any function of $K_{123}$, and thus will give no contribution from acting on the $K_{1 \cdots c_{2}}$ inside the cut (6.17). The only action left is that on the lone $\bar{\lambda}_{1} \mathrm{~s}$ in $D$, that is, on

$$
2 \pi i c_{\Gamma} A^{\text {tree }} \ln \left(\frac{\left\langle\lambda_{c_{2}+1}{ }^{-}\left|K_{1 \cdots c_{2}}\right| \bar{\lambda}_{1}^{-}\right\rangle}{\left\langle\lambda_{c_{2}}{ }^{-}\left|\not K_{1 \cdots c_{2}}\right| \bar{\lambda}_{1}^{-}\right\rangle}\right)
$$


computing that, we find

$$
\eta^{\dot{a}} F_{123 \dot{a}} D=2 \pi i\left\langle\lambda_{2}, \lambda_{3}\right\rangle c_{\Gamma} A^{\text {tree }}\left[\frac{\left\langle\lambda_{c_{2}+1}{ }^{-}\left|K_{1 \cdots c_{2}}\right| \bar{\eta}^{-}\right\rangle}{\left\langle\lambda_{c_{2}+1^{-}}\left|K_{1 \cdots c_{2}}\right| \bar{\lambda}_{1}^{-}\right\rangle}-\frac{\left\langle\lambda_{c_{2}}{ }^{-}\left|K_{1 \cdots c_{2}}\right| \bar{\eta}^{-}\right\rangle}{\left\langle\lambda_{c_{2}}{ }^{-}\left|K_{1 \cdots c_{2}}\right| \bar{\lambda}_{1}{ }^{-}\right\rangle}\right] .
$$

Using the Schouten identity, we can simplify this to

$$
2 \pi i\left\langle\lambda_{2}, \lambda_{3}\right\rangle c_{\Gamma} A^{\text {tree }} \frac{s_{1 \cdots c_{2}}\left[\bar{\eta}, \bar{\lambda}_{1}\right]\left\langle\lambda_{c_{2}}, \lambda_{c_{2}+1}\right\rangle}{\left\langle\lambda_{c_{2}}{ }^{-}\left|\not K_{1 \cdots c_{2}}\right| \bar{\lambda}_{1}^{-}\right\rangle\left\langle\lambda_{c_{2}+1}\left|\not K_{1 \cdots c_{2}}\right| \bar{\lambda}_{1}^{-}\right\rangle}
$$

so that

$$
F_{123}^{\prime} D=0
$$

Cut constructibility then implies that $F_{123}^{\prime}$ also annihilates the dispersive parts of this diagram.

\section{Conclusions}

Tree-level amplitudes in twistor space have a simple structure. They can be obtained from correlation functions evaluated on disconnected $D$-instantons of degree one, linked by propagators. Starting from the BST construction of one-loop amplitudes based on MHV vertices, we have shown that one-loop MHV amplitudes have a similar simple structure. They can also be obtained from correlation functions evaluated on disconnected degree-one instantons, in this case linked by two propagators. This suggests that more general one-loop amplitudes should have a similar structure. In particular, the discussion here, along with earlier predictions by Dixon [24] and also the picture emerging from NMHV loop calculations by Dixon, Del Duca and two of the authors [25], suggests that the one-loop next-to-MHV amplitudes should be obtained from the configurations shown in fig. 4. We have considered the $\mathcal{N}=4$ supersymmetric gauge theory in this paper, but it is clear that the arguments apply equally well to any supersymmetric theory, because the four-dimensional cuts suffice to construct the full amplitude [19].

We have also shown that the infrared divergences characteristic of loop amplitudes in gauge theories are isolated in a certain class of twistor-space diagrams. This means that the question of how to derive generic diagrams from a twistor-space string theory can be separated from the issue of infrared divergences. We have also shown how to introduce an infrared regulator corresponding to a momentum-space dimensional regulator for the subset of diagrams that do have infrared divergences.

We have evaluated the CSW 'holomorphic anomaly' explicitly, and shown that it can be used

to define modified differential operators whose kernels contain the one-loop MHV amplitudes in agreement with the structure outlined above. 

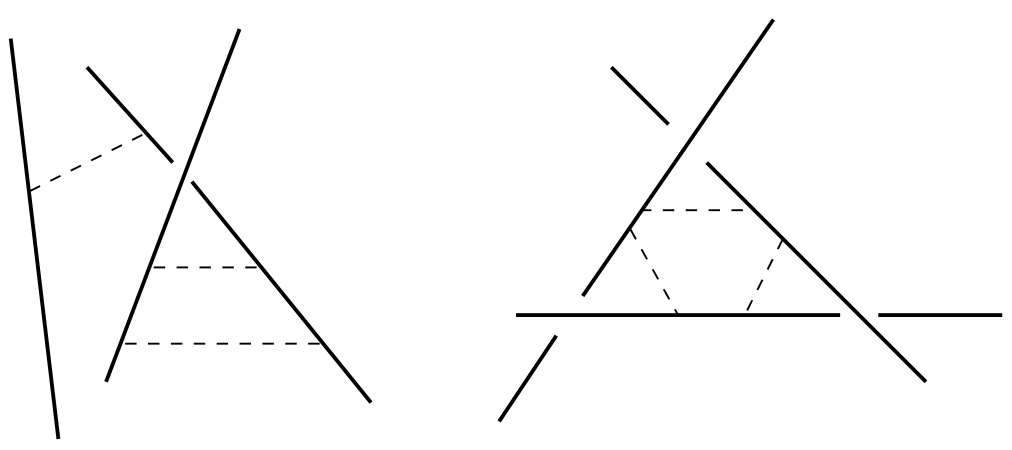

Figure 4. The twistor-space configurations expected to contribute to a one-loop NMHV amplitude, in accordance with earlier predictions by Dixon [24] and as motivated by NMHV one-loop calculations by Dixon, Del Duca and two of the authors [25].

One may wonder to what extent an equivalence can exist between this twistor-space picture and one in which the one-loop amplitudes are supported on a lone higher-degree genus-one curve. At the very least, one would need a method of projecting out the contributions of the superconformal gravitons to the amplitude. In the translation of the BST construction we have performed in this paper, this projection is implemented by restricting the propagators to be gauge-multiplet propagators.

We thank L. J. Dixon and V. P. Nair for useful conversations, and the Kavli Institute for Theoretical Physics, where this work was carried out, for its generous hospitality. This research was supported in part by the National Science Foundation under grants PHY99-07949, PHY00-98395, PHY00-99590 and PHY01-40151 and by the US Department of Energy under grants DE-FG0291ER40671, DE-FG02-91ER40618 and DE-FG03-91ER40662.

\section{Appendix I. Fourier Transforming for Dimensional Regularization}

In order to introduce an appropriate dimensional regulator for infrared-divergent diagrams, we need to evaluate the Fourier transform of $\left[\tilde{\lambda}_{a}, \tilde{\lambda}_{b}\right]^{-\epsilon}$, which we do in this appendix.

The required transform is,

$$
\begin{aligned}
& \int \frac{d^{2} \tilde{\lambda}_{a, b}}{(2 \pi)^{4}}\left(\tilde{\lambda}_{a}^{i} \tilde{\lambda}_{b}^{\dot{2}}-\tilde{\lambda}_{a}^{2} \tilde{\lambda}_{b}^{\dot{1}}-i \eta^{\prime}\right)^{-\epsilon} e^{i\left(\mu_{a}^{\mathrm{i}} \tilde{\lambda}_{a}^{2}-\mu_{a}^{2} \tilde{\lambda}_{a}^{\mathrm{i}}+\mu_{b}^{\mathrm{i}} \tilde{\lambda}_{b}^{\dot{2}}-\mu_{b}^{2} \tilde{\lambda}_{b}^{\mathrm{i}}\right)} \\
& =\int \frac{d^{2} \tilde{\lambda}_{a, b}}{(2 \pi)^{4}}\left(-i \tilde{\lambda}_{b}^{\dot{2}}\right)^{-\epsilon}\left(i \tilde{\lambda}_{a}^{\dot{1}}-i \frac{\tilde{\lambda}_{a}^{\dot{2}} \tilde{\lambda}_{b}^{\mathrm{i}}}{\tilde{\lambda}_{b}^{\dot{2}}}+\eta\right)^{-\epsilon} e^{i\left(\mu_{a}^{\mathrm{i}} \tilde{\lambda}_{a}^{\dot{2}}-\mu_{a}^{\dot{2}} \tilde{\lambda}_{a}^{\dot{1}}+\mu_{b}^{\mathrm{i}} \tilde{\lambda}_{b}^{\dot{2}}-\mu_{b}^{2} \tilde{\lambda}_{b}^{\mathrm{i}}\right)},
\end{aligned}
$$


where $\eta^{\prime}=\eta \tilde{\lambda}_{b}^{\dot{2}}$ is a regulator.

We use the formula (eq. (3.2.4) of ref. [26])

$$
\int(a+i x)^{-\epsilon} e^{-i k x}=-\frac{2 \pi}{\Gamma(\epsilon)}(-k)^{\epsilon-1} e^{a k},
$$

to do the $\tilde{\lambda}_{a}^{\mathrm{i}}$ integral,

$$
-\int \frac{d^{2} \tilde{\lambda}_{b} d \tilde{\lambda}_{a}^{\dot{2}}}{(2 \pi)^{4}} \frac{2 \pi}{\Gamma(\epsilon)}\left(-i \tilde{\lambda}_{b}^{\dot{2}}\right)^{-\epsilon} e^{i\left(\mu_{a}^{\mathrm{i}} \tilde{\lambda}_{a}^{\dot{2}}+\mu_{b}^{\mathrm{i}} \tilde{\lambda}_{b}^{\dot{2}}-\mu_{b}^{\dot{2}} \tilde{\lambda}_{b}^{\dot{1}}\right)}\left(-\mu_{a}^{\dot{2}}\right)^{\epsilon-1} \exp \left[-i \mu_{a}^{2} \frac{\tilde{\lambda}_{a}^{\dot{2}} \tilde{\lambda}_{b}^{\dot{1}}}{\tilde{\lambda}_{b}^{\dot{2}}}+\mu_{a}^{\dot{2}} \eta\right] .
$$

Next, do the $\tilde{\lambda}_{a}^{\dot{2}}$ integral,

$$
\begin{aligned}
& -\frac{\epsilon}{(2 \pi)^{2} \Gamma(1+\epsilon)} \int d^{2} \tilde{\lambda}_{b}\left(-i \tilde{\lambda}_{b}^{\dot{2}}\right)^{-\epsilon} e^{i\left(\mu_{b}^{i} \tilde{\lambda}_{b}^{\dot{2}}-\mu_{b}^{2} \tilde{\lambda}_{b}^{\mathrm{i}}\right)}\left(-\mu_{a}^{\dot{2}}\right)^{\epsilon-1} \exp \left[\mu_{a}^{\dot{2}} \eta\right] \delta\left(\mu_{a}^{\dot{1}}-\frac{\mu_{a}^{\dot{2}} \tilde{\lambda}_{b}^{\dot{1}}}{\tilde{\lambda}_{b}^{\dot{2}}}\right) \\
& =-\frac{\epsilon}{(2 \pi)^{2} \Gamma(1+\epsilon)} \int d \tilde{\lambda}_{b}^{\dot{2}}\left(-i \tilde{\lambda}_{b}^{\dot{2}}\right)^{-\epsilon}\left(-\mu_{a}^{\dot{2}}\right)^{\epsilon-1} \exp \left[\mu_{a}^{\dot{2}} \eta^{\prime} / \tilde{\lambda}_{b}^{\dot{2}}\right]\left(-\mu_{a}^{\dot{2}}\right)^{-1}\left(-\tilde{\lambda}_{b}^{\dot{2}}\right) \exp \left[i\left(\mu_{b}^{\dot{1}} \tilde{\lambda}_{b}^{\dot{2}}-\frac{\mu_{b}^{\dot{2}} \mu_{a}^{\dot{1}} \tilde{\lambda}_{b}^{\dot{2}}}{\mu_{a}^{\dot{2}}}\right)\right] .
\end{aligned}
$$

Only the sign of the coefficient of $\eta$ inside the exponential matters, so we can rewrite it as $\exp \left[\eta^{\prime} \tilde{\lambda}_{b}^{\dot{2}} / \mu_{a}^{\dot{2}}\right]$. To do the last Fourier transform we use the inverse of eq. (I.2), with $\epsilon^{\prime}=2-\epsilon$, obtaining

$$
\begin{aligned}
& (i)^{-\epsilon} \frac{\epsilon \Gamma(2-\epsilon)}{(2 \pi)^{3} \Gamma(1+\epsilon)}\left(-\mu_{a}^{\dot{2}}\right)^{\epsilon-2}\left(i\left(\mu_{b}^{\dot{1}}-\frac{\mu_{b}^{2} \mu_{a}^{\dot{1}}}{\mu_{a}^{\dot{2}}}\right)+\eta^{\prime} / \mu_{a}^{\dot{2}}\right)^{\epsilon-2} \\
& =\frac{\epsilon \Gamma(2-\epsilon)}{(2 \pi)^{3} \Gamma(1+\epsilon)}\left(\mu_{b}^{\dot{2}} \mu_{a}^{\dot{1}}-\mu_{b}^{\dot{1}} \mu_{a}^{\dot{2}}+i \eta^{\prime}\right)^{\epsilon-2} \\
& =\frac{\epsilon \Gamma(2-\epsilon)}{(2 \pi)^{3} \Gamma(1+\epsilon)}\left[\mu_{a}, \mu_{b}\right]^{\epsilon-2} .
\end{aligned}
$$

As $\epsilon \rightarrow 0$ this turns into a product of $\delta$ functions for all four components of the $\mu \mathrm{s}$. We can therefore regard it as defining a delta-convergent sequence, or equivalently a smearing of the twistor-space delta function.

\section{Appendix II. Evaluation of the Jacobian}

In this appendix we compute the jacobian appearing in eq. (6.10), arising from the phase space integration over the anomaly-induced delta functions in eq. (6.3). The jacobian is

$$
\mathcal{J}=\int \frac{d^{4} \ell_{1} \delta^{(+)}\left(\ell_{1}^{2}\right)}{(2 \pi)^{3}} \frac{d^{4} \ell_{2} \delta^{(+)}\left(\ell_{2}^{2}\right)}{(2 \pi)^{3}}(2 \pi)^{4} \delta^{4}\left(K_{1 \cdots c_{2}}-\ell_{1}-\ell_{2}\right) \delta\left(\left\langle\lambda_{\ell_{1}}, \lambda_{\ell_{2}}\right\rangle\right) \delta\left(\left[\bar{\lambda}_{\ell_{1}}, \bar{\lambda}_{\ell_{2}}\right]\right) .
$$

Expressing the phase space integral in terms of the CSW measure given in eq. (2.16) yields,

$$
\begin{aligned}
\mathcal{J}=c_{\Gamma} & \int_{0}^{\infty} d T_{1} \int_{0}^{\infty} d T_{2} \int_{\mathbb{C}} d w_{1} d \bar{w}_{1} \int_{\mathbb{C}} d w_{2} d \bar{w}_{2} T_{2} \delta\left(\lambda_{1}^{2}-\lambda_{1}^{1} w_{1}\right) \delta\left(\bar{\lambda}_{1}^{\dot{2}}-\bar{\lambda}_{1}^{\dot{1}} \bar{w}_{1}\right) \\
& \times \delta^{4}\left(\left(\begin{array}{cc}
K_{1 \cdots c_{2}}^{0}+K_{1 \cdots c_{2}}^{3} & K_{1 \cdots c_{2}}^{1}-i K_{1 \cdots c_{2}}^{2} \\
K_{1 \cdots c_{2}}^{1}+i K_{1 \cdots c_{2}}^{2} & K_{1 \cdots c_{2}}^{0}-K_{1 \cdots c_{2}}^{3}
\end{array}\right)-T_{1}\left(\begin{array}{cc}
1 & \bar{w}_{1} \\
w_{1} & w_{1} \bar{w}_{1}
\end{array}\right)-T_{2}\left(\begin{array}{cc}
1 & \bar{w}_{2} \\
w_{2} & w_{2} \bar{w}_{2}
\end{array}\right)\right),
\end{aligned}
$$


where we have set $T_{1}=\tau_{1}^{2}$ and $T_{2}=\tau_{2}^{2}$. (In this expression, $\epsilon$ in the $c_{\Gamma}$ factor is set to zero since here we consider infrared finite diagrams.) The $\delta$-functions yield a set of equations,

$$
\begin{aligned}
& f_{1} \equiv \lambda_{1}^{2}-\lambda_{1}^{1} w_{1}=0 \\
& f_{2} \equiv \bar{\lambda}_{1}^{2}-\bar{w}_{1} \bar{\lambda}_{1}^{\mathrm{i}}=0, \\
& f_{3} \equiv-T_{2} w_{2}-T_{1} w_{1}+\left(K_{1 \cdots c_{2}}^{1}+i K_{1 \cdots c_{2}}^{2}\right)=0, \\
& f_{4} \equiv-T_{2} \bar{w}_{2}-T_{1} \bar{w}_{1}+\left(K_{1 \cdots c_{2}}^{1}-i K_{1 \cdots c_{2}}^{2}\right)=0, \\
& f_{5} \equiv-T_{1}-T_{2}+\left(K_{1 \cdots c_{2}}^{0}+K_{1 \cdots c_{2}}^{3}\right)=0, \\
& f_{6} \equiv-T_{1} w_{1} \bar{w}_{1}-T_{2} w_{2} \bar{w}_{2}+\left(K_{1 \cdots c_{2}}^{0}-K_{1 \cdots c_{2}}^{3}\right)=0 .
\end{aligned}
$$

These equation are all independent and hence fix all integration variables. Integrating the over the delta function leads to the jacobian

$$
\begin{aligned}
\mathcal{J} & =c_{\Gamma} T_{2}\left|\frac{\partial\left(f_{1}, f_{2}, f_{3}, f_{4}, f_{5}, f_{6}\right)}{\partial\left(w_{1}, w_{2}, \bar{w}_{1}, \bar{w}_{2}, T_{1}, T_{2}\right)}\right|^{-1} \\
& =c_{\Gamma}\left[T_{2} \lambda_{1}^{1} \bar{\lambda}_{1}^{i}\left(w_{2}-w_{1}\right)\left(\bar{w}_{2}-\bar{w}_{1}\right)\right]^{-1} \\
& =c_{\Gamma} \frac{1}{2 k_{1} \cdot K_{1 \cdots c_{2}}},
\end{aligned}
$$

where we substituted the solution to the relations (II.3) to obtain the result on the last line. This jacobian factor is independent of the particular one-loop cut amplitude under consideration and applies just as well to the non-MHV case. 


\section{References}

[1] E. Witten, hep-th/0312171.

[2] V. P. Nair, Phys. Lett. B214:215 (1988).

[3] S. J. Parke and T. R. Taylor, Phys. Rev. Lett. 56:2459 (1986).

[4] F. A. Berends and W. T. Giele, Nucl. Phys. B306:759 (1988).

[5] F. Cachazo, P. Svrček and E. Witten, hep-th/0403047.

[6] G. Georgiou and V. V. Khoze, JHEP 0405:070 (2004) hep-th/0404072;

J. B. Wu and C. J. Zhu, JHEP 0407:032 (2004) hep-th/0406085;

J. B. Wu and C. J. Zhu, hep-th/0406146;

G. Georgiou, E. W. N. Glover and V. V. Khoze, JHEP 0407:048 (2004) hep-th/0407027;

V. V. Khoze, hep-th/0408233;

M. Lou and C. Wen, hep-th/0410045.

[7] I. Bena, Z. Bern and D. A. Kosower, hep-th/0406133.

[8] D. A. Kosower, hep-th/0406175.

[9] N. Berkovits, hep-th/0402045;

N. Berkovits and L. Motl, JHEP 0404:056 (2004) hep-th/0403187.

[10] A. Neitzke and C. Vafa, hep-th/0402128.

[11] W. Siegel, hep-th/0404255.

[12] A. Brandhuber, B. Spence and G. Travaglini, hep-th/0407214.

[13] Z. Bern, L. J. Dixon, D. C. Dunbar, and D. A. Kosower, Nucl. Phys. B425:217 (1994) hep$\mathrm{ph} / 9403226]$.

[14] Y. Abe, V. P. Nair and M. I. Park, hep-th/0408191.

[15] S. Gukov, L. Motl and A. Neitzke, hep-th/0404085.

[16] R. Roiban, M. Spradlin and A. Volovich, JHEP 0404:012 (2004) hep-th/0402016;

R. Roiban and A. Volovich, hep-th/0402121;

R. Roiban, M. Spradlin and A. Volovich, hep-th/0403190.

[17] F. Cachazo, P. Svrček and E. Witten, hep-th/0406177.

[18] F. Cachazo, P. Svrček and E. Witten, hep-th/0409245.

[19] Z. Bern, L. J. Dixon, D. C. Dunbar and D. A. Kosower, Nucl. Phys. B435:59 (1995) hep$\mathrm{ph} / 9409265]$.

[20] Z. Bern, L. J. Dixon and D. A. Kosower, Nucl. Phys. Proc. Suppl. 51C:243 (1996) hepph/9606378];

Z. Bern, L. J. Dixon and D. A. Kosower, Ann. Rev. Nucl. Part. Sci. 46:109 (1996) hepph/9602280; 
Z. Bern and A. G. Morgan, Nucl. Phys. B467:479 (1996) hep-ph/9511336;

Z. Bern, L. J. Dixon and D. A. Kosower, JHEP 0408:012 (2004) hep-ph/0404293.

[21] Z. Kunszt, A. Signer and Z. Trocsanyi, Nucl. Phys. B420:550 (1994) hep-ph/9401294.

[22] D. A. Kosower, unpublished.

[23] Z. Bern, L. J. Dixon and D. A. Kosower, Nucl. Phys. B513:3 (1998) hep-ph/9708239.

[24] L. J. Dixon, private communication.

[25] Z. Bern, V. Del Duca, L. J. Dixon and D. A. Kosower, in progress.

[26] A. Erdélyi et. al., Tables of Integral Transforms, Vol I, (McGraw-Hill, 1954). 